\title{
Electron bunch compression using a laser-plasma compressor
}

\author{
W. van Dijk, ${ }^{*}$ M. J. van der Wiel, and G. J.H. Brussaard \\ Center for Plasma Physics and Radiation Technology, Eindhoven University of Technology, P.O. Box 513, 5600 MB Eindhoven, \\ The Netherlands
}

(Received 23 June 2009; published 12 November 2009)

\begin{abstract}
A new scheme for the compression of electron bunches is proposed. This scheme uses a plasma wave generated by a high intensity laser pulse to compress an electron bunch produced by a typical rf photogun. In the scheme, the electron bunch is injected into a plasma channel in front of the high intensity laser pulse. The laser pulse generates a plasma wave which "sweeps up" the electron bunch resulting in both compression and acceleration. The electron bunch will eventually overtake the laser pulse so that, afterwards, the electrons travel in front of the plasma wave. The scheme differs from previous schemes using a plasma wave to accelerate and compress electron bunches by sacrificing part of the acceleration to get a lower final energy spread and decrease the sensitivity to jitter in the synchronization between laser and electrons. Using a hybrid model, combining particle tracking for the electrons and a relativistic fluid model for the plasma, the scheme is investigated. The model is used to study the effects of the size of the injected bunches, the initial energy spread, and jitter in the laser-bunch synchronization. Also, one of the ways to vary the compression ratio of the scheme is explored by varying the plasma density. From these simulations, it can be concluded that the proposed scheme can compress bunches from an initial size of picoseconds to below one hundred femtoseconds with an energy spread below $0.2 \%$. The scheme can also be adjusted to still produce these bunches in the presence of synchronization jitter up to 1 ps (for the parameters chosen).
\end{abstract}

DOI: 10.1103/PhysRevSTAB.12.111302

PACS numbers: 41.85.Ct, 41.75.Jv, 52.59.-f, 29.25.Bx

\section{INTRODUCTION}

The generation of sub-100-fs electron bunches enables production of coherent $\mathrm{THz}$ radiation [1] and coherent $\mathrm{x}$-ray radiation [2] as well as a variety of pump-probetype experiments on these short time scales. However, most sources of electron bunches produce bunches which are considerably longer than 100 fs which means these bunches either have to be compressed or chopped to create shorter (sub)bunches. Since most applications also benefit from high peak currents, compression is often preferred. The most common method of compression uses a magnetic bunch compression chicane [3-5]. The off-axis displacement of the electrons needed for this compression scheme can however lead to instabilities [6] and phase-space distortions [7]. Also, the length of the chicanes needed for compression can be several tens of meters or more long.

Laser wakefield acceleration [8] uses the electric fields generated by an intense laser pulse in a plasma to accelerate charged particles in fields that are orders of magnitude higher than those encountered in conventional accelerators. Experiments have thus far been able to generate bunches at energy of 100's of MeV [9-11] and higher [12] (up to $1 \mathrm{GeV}$ ) with very short duration (5-10 fs).

\footnotetext{
*w.v.dijk@tue.nl
}

This paper will focus on the ability of laser wakefield accelerators to create short electron bunches. Specifically, we will look at using a plasma wave to reduce the length of a picosecond electron bunch, which can be produced in a conventional rf accelerator to the sub-10-fs level while keeping the energy spread low. Previous schemes for injecting electrons both behind [13-16] and in front [17-19] of the laser pulse predict the generation of sub-10-fs electron bunches. However, in these schemes timing jitter and the energy spread present within these bunches can quickly lead to bunch lengthening. The scheme presented in this paper, dubbed the "plasma compressor," uses a technique similar to the acceleration scheme presented in $[17,18]$ but sacrifices a large part of the energy gain to reduce the energy spread, decrease the sensitivity to timing variations [20], and achieve strong compression for injected bunches that are longer than those described in $[17,18]$.

With this added reliability, the plasma compressor can be used as a module in an otherwise conventional accelerator setup to create a beam line producing ultrashort electron bunches.

\section{THEORY}

\section{Process description}

The concept of the compression scheme is depicted in Fig. 1. First, a relatively long electron bunch is injected in 

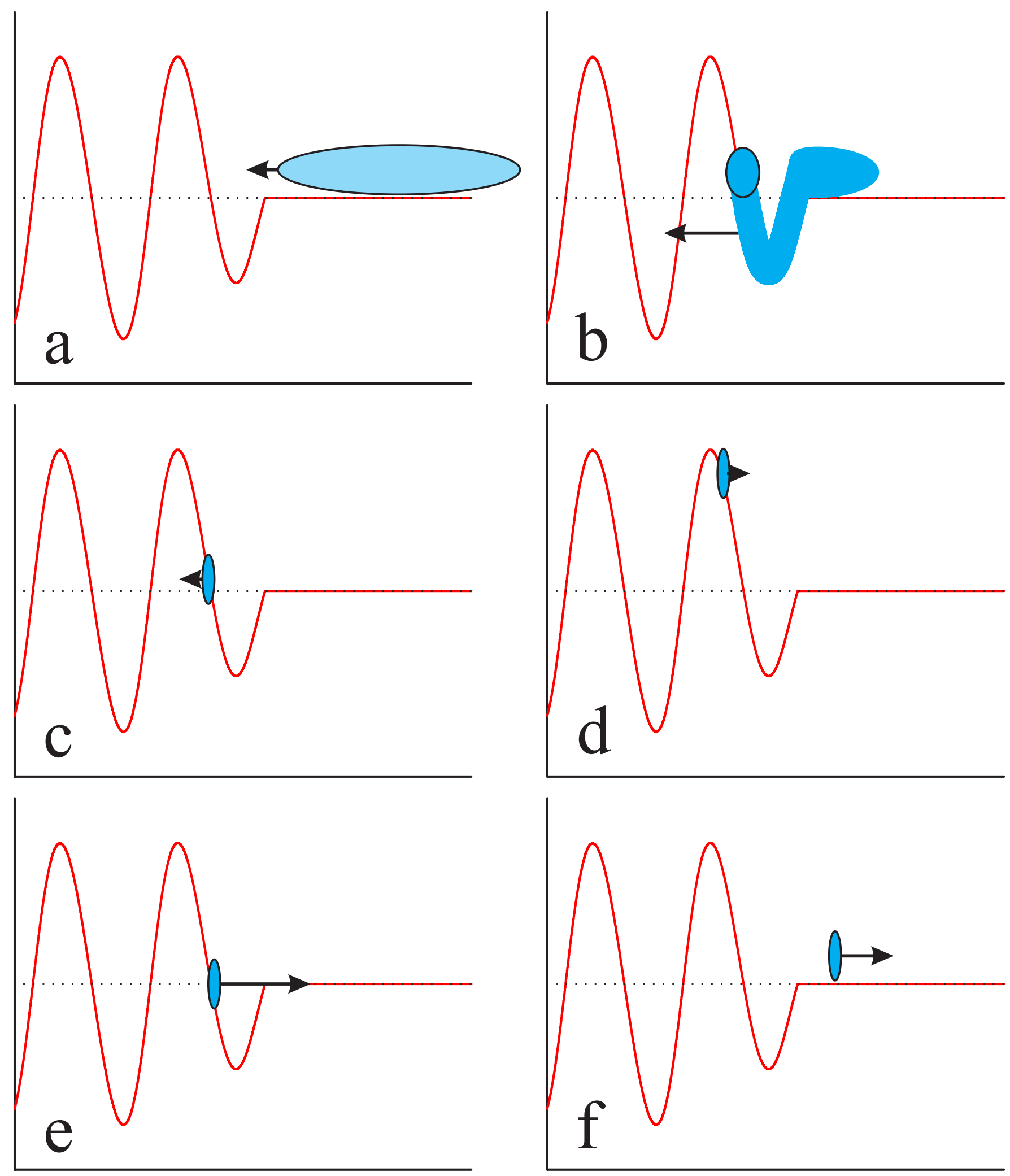

FIG. 1. (Color) Schematic representation of the plasma-compressor scheme in the calculated fields (see model in Sec. III) of the plasma wave: (a) the electron bunch just before the plasma wave overtakes it; (b) the rear part of the electron bunch is decelerated by the front of the plasma wave and gets compressed; (c) the electron bunch is compressed and gets accelerated; (d) the electrons start outpacing the plasma wave while still being accelerated; (e) the electrons get decelerated again by the plasma wave; (f) the compressed bunch leaves the plasma wave. 
front of a laser generated plasma wave [Fig. 1(a)] as proposed by Khachatryan [17,18,21]. If the electron bunch energy and laser power are sufficient, the plasma wave will first decelerate [Fig. 1(b)] and then accelerate and trap the electrons. During this trapping and acceleration, the electron bunch is compressed [Fig. 1(c)]. The compressed bunch is then accelerated to a velocity greater than that of the plasma wave and starts outpacing the plasma wave [Fig. 1(d)] until it reaches the decelerating phase of the plasma wave [Fig. 1(e)] (dephasing). At this point the electron bunch is short, has a high energy, and depending on the initial electron energy and bunch length a low to moderate energy spread. This is where the plasma is terminated in the scheme proposed by Khachatryan. In the scheme proposed in this paper, the electron bunch is allowed to pass through the decelerating phase of the plasma wave and emerge in front of the plasma wave [Fig. 1(f)]. This results in a short electron bunch with a lower energy, but with a much lower energy spread.

Because all electrons of the injected bunch pass through the same phases of the plasma wave (starting and ending before the laser pulse), they will all experience the same electric fields and therefore retain their low energy spread. There is still a net acceleration in this scheme since the decelerating field at the front of the plasma wave is less strong than the accelerating field just behind it, where the electrons are initially trapped and accelerated (as can be schematically seen in Fig. 1).

In order to estimate the amount of compression, we can use the 1D theoretical approximation for the compressed bunch length $L_{c}$ of a monoenergetic bunch by Khachatryan et al. [17]:

$$
L_{c} \simeq\left(\frac{\gamma_{0}}{\gamma_{g}}\right)^{2} L_{0}
$$

with $\gamma_{0}$ the Lorentz factor for the injected electrons, $\gamma_{g}$ the Lorentz factor for the plasma wave, and $L_{o}$ the length of the original electron bunch. After the trapping and compression [Fig. 1(c)], the bunch length is small compared to the variations in the electric fields (in the frame of the electrons) which means there is no (almost) net bunch lengthening by the plasma wave once the bunch has left the plasma wave [Fig. 1(f)]. There might, however, still be additional bunch lengthening due to initial energy spread and space charge in the bunch, both of which will be looked at later.

\section{MODEL}

The model used is the same as previously used to study the effects of timing and stability in injected laser wakefield acceleration [20]. It consists of a system of relativistic hydrodynamic and Maxwell's equations describing the plasma wave and a particle tracking framework for the accelerated electrons. By assuming the laser is guided [22-25] and neglecting the effects of beam loading and
TABLE I. General parameters of plasma, laser, and electron bunches.

\begin{tabular}{lcc}
\hline \hline Plasma & Density $\left[\mathrm{m}^{-3}\right]$ & $1.0 \times 10^{24}$ \\
& Radius $[\mu \mathrm{m}]$ & 150 \\
& Length [mm] & $326^{\mathrm{a}}$ \\
& Matched spot size $(\mathrm{FWHM})[\mu \mathrm{m}]$ & 52 \\
\hline Laser & Pulse energy $[\mathrm{mJ}]$ & 1500 \\
& Pulse duration $(\mathrm{FWHM})[\mathrm{fs}]$ & 50 \\
& Pulse $a_{o}$ & 0.56 \\
Bunch & Pulse delay [fs] & 1500 \\
& Energy [MeV] & 4.6 \\
& $\gamma$ & 10 \\
& Charge $[\mathrm{pC}]$ & 10 \\
& Radial size (FWHM) $[\mu \mathrm{m}]$ & 30 \\
Length $(\mathrm{FWHM})[\mathrm{fs}]$ & 500 \\
\hline \hline
\end{tabular}

${ }^{\text {a }}$ Plasma length increased by $\sim 40 \%$ to increase stability against timing fluctuations and allow for longer bunches (needed for increased laser pulse delay and extended plasma length beyond full dephasing length).

laser pulse evolution (which can be done for the parameters considered (Table I), we can use a local, comoving description of the plasma wave. This greatly reduces the amount of computation power needed and allows multiple calculations involving long (several $\mathrm{cm}$ ) plasma channels on a regular desktop computer during a day.

In order to describe the plasma wave, we use a system of relativistic hydrodynamic equations of motion for the plasma electrons, the Maxwell equations and a beam envelope description of the laser pulse in an approach analogous to $[26,27]$. This approach results in the following set of equations for the plasma evolution:

$$
\begin{gathered}
\frac{\partial n}{\partial t}+\vec{\nabla} \cdot(n \vec{v})=0, \\
\frac{\partial \vec{p}}{\partial t}=e \vec{E}-m_{0} c^{2} \vec{\nabla} \gamma, \\
\frac{\partial \vec{E}}{\partial t}=-4 \pi e n \vec{v}-\frac{c^{2}}{e} \vec{\nabla} \times(\vec{\nabla} \times \vec{p}), \\
\gamma=\sqrt{1+\frac{|\vec{p}|^{2}}{m_{0}^{2} c^{2}}+\frac{|\vec{a}|^{2}}{2},}
\end{gathered}
$$

$$
\vec{v}=\frac{\vec{p}}{m_{0} \gamma}
$$

Here $e$ is the charge of an electron, $m_{0}$ the electron rest mass, and $c$ the speed of light. The plasma electrons are described by their density $n$, momentum $\vec{p}$, velocity $\vec{v}$, and Lorentz factor $\gamma$. The electric field strength is denoted by $\vec{E}$ and the normalized vector potential of the laser by $\vec{a}$ : 


$$
\vec{a}=\frac{e \vec{A}}{m_{0} c}
$$

with $\vec{A}$ the vector potential of the laser pulse.

These equations are evaluated in a frame that is comoving with the laser pulse as described in [20].

To study the effect of the plasma wave on the electron bunch, we use the general particle tracker code [28]. The code allows the inclusion of custom elements such as the plasma wave module and evaluations of (custom) electron bunch parameters.

The model used does not include the effects of beam loading and space charge on the compression. As the electron bunch is compressed more and more, these two effects will start to reduce the rate of compression. To properly study the results of these effects, it is necessary to include the effects of the electron bunch on the plasma electrons and the effect of the plasma on the interactions between the electrons in the bunch. These effects are
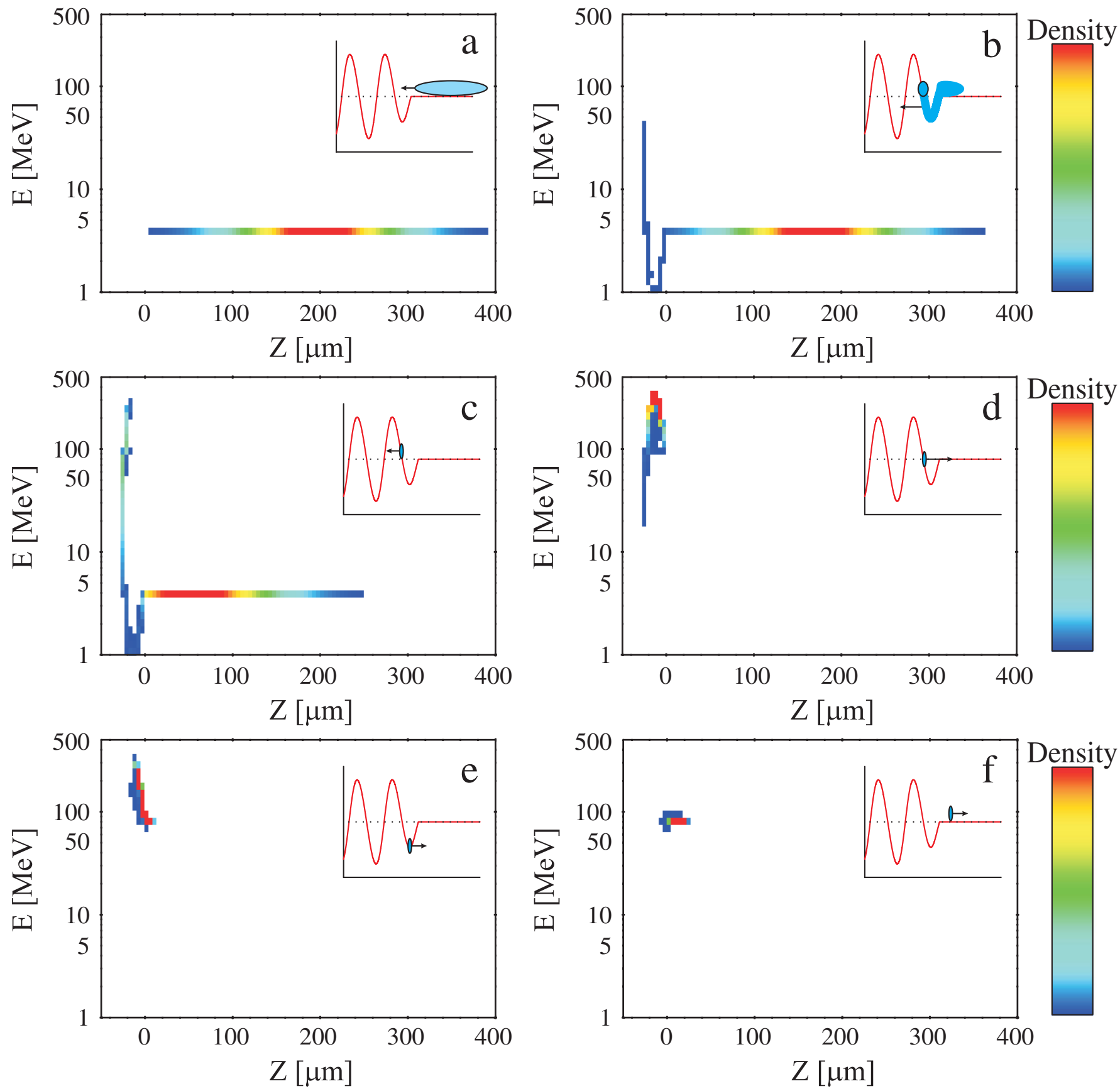

FIG. 2. (Color) Density plots of the simulation results for the energy and bunch length evolution during compression of a 0.5 ps electron bunch. The insets are the equivalent situations from Fig. 1. 
included in several particle in cell codes, but these codes need an unpractical amount of computational power and time to simulate plasma channels such as the one used in this paper. It is however possible to include the effects of space charge at the cost of increasing the necessary computational power; this will be discussed in Sec. IV F.

\section{Standard parameters}

In order to study the compression scheme, we will first give the relevant base parameters of the plasma, laser, and uncompressed electron bunch used. These can be found in Table I. The plasma is assumed to be a slow capillary discharge plasma [29] and capable of guiding a laser with matched spot size as calculated from [30]. The injected electron bunch energy is the same as that which provides maximum energy and minimum energy spread when using the plasma wave for acceleration.

As can be seen from Table I, the plasma length and laser pulse delay are set at a large value to allow the use of long injected bunches (Sec. IV D) and to improve the stability against timing jitter (Sec. IV B). If the timing jitter and bunch length are smaller, both the plasma length and laser pulse delay can be reduced.

\section{SIMULATION RESULTS}

\section{A. beam evolution}

First, we will look at the time evolution of a bunch injected in front of the laser pulse in a plasma using the parameters given in Table I and compare it to the schematic representation of Fig. 1. The result is shown in Fig. 2.

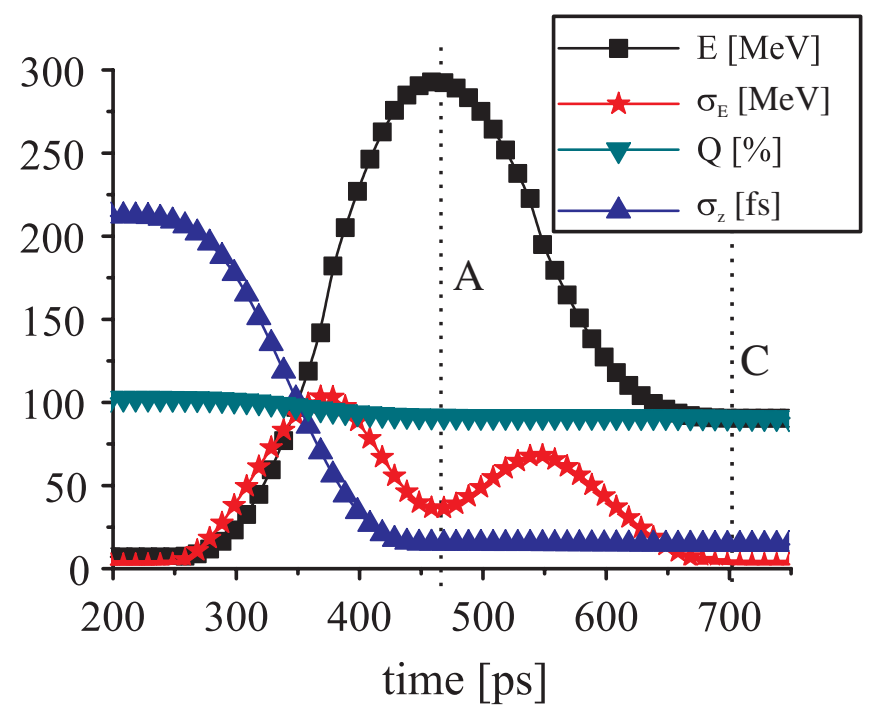

FIG. 3. (Color) Time evolution of the energy $(E)$, energy spread $\left(\sigma_{E}\right)$, charge $(Q)$, and bunch length $\left(\sigma_{z}\right)$. The first dotted line (A) denotes the end of the plasma in the accelerator scheme while the second dotted line $(\mathrm{C})$ indicates the end of the plasma in the new compression scheme.
TABLE II. Properties of the electron bunch before injection and when exiting the plasma in the accelerator and compressor schemes.

\begin{tabular}{lccc}
\hline \hline Parameter & Injected & Accelerated & Compressed \\
\hline Energy $[\mathrm{MeV}]$ & 4.6 & 290 & 88 \\
Energy spread $[\mathrm{MeV}]$ & 0 & 34 & 0.19 \\
Charge $[\%]$ & 100 & 89 & 88 \\
Length $\left(\sigma_{z}\right)[\mathrm{fs}]$ & 210 & 13 & 12 \\
\hline \hline
\end{tabular}

As can be seen from Fig. 2, the simulations and the schematic representation roughly agree, but in the simulations there is an overlap between the different phases of the scheme because of the length of the injected bunch: In Fig. 2(c) part of the bunch is still in front of the wakefield while another part is already trapped and being accelerated. A bit later [Fig. 2(d)], one end of the bunch is just entering the accelerating phase of the plasma wave, while the other is already leaving the decelerating phase. To better evaluate the effect of the compression, we will look at the time evolution of the energy, energy spread, the remaining bunch charge, and the bunch length. These can be found in Fig. 3.

In Fig. 3 we have denoted the moment of bunch extraction in the case of the original accelerator scheme (A) and the compression scheme proposed here $(\mathrm{C})$. As can be seen, the trapped bunch charge and bunch length are the same, but in the case of the compression scheme, the energy spread is significantly lower at the cost of the average energy. The parameters of the electron bunch before injection and in the two schemes can be found in Table II.

The change in emittance in both the transverse and longitudinal direction of the bunch during compression is shown in Fig. 4. During compression, different parts of the

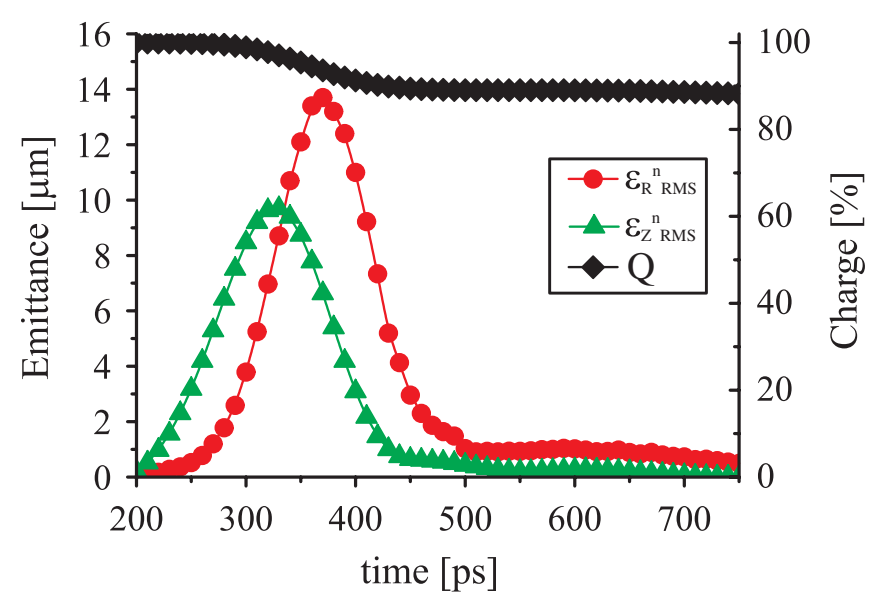

FIG. 4. (Color) Time evolution of the normalized RMS emittance in the transverse direction $\left(\varepsilon_{R_{\mathrm{RMS}}}^{N}\right)$ and the longitudinal direction $\left(\varepsilon_{Z_{\mathrm{RMS}}}^{N}\right)$ as well as the total amount or charge left in the bunch $(Q)$. 
bunch experience different forces in the transverse (focusing) and longitudinal (accelerating/decelerating) direction, this leads to a nonuniform distortion of the phase space and corresponding increase in emittance (though the actual local phase-space density remains unchanged). During entire compression, the different parts of the bunch all experience (almost) the same forces (just at different times); this means that after compression, the emittance goes down again until both are below $1 \mu \mathrm{m}[\mathrm{rad}]$. Since not all parts of the bunch experience exactly the same forces, there is still a remaining distortion leading the increased final emittance. The lowering of the emittance is enhanced by the removal of the radially expelled electrons when they hit the radial plasma boundary. As outliers in phase space, these electrons have a strong influence on the emittance and as they are removed in time, the total emittance decreases.

\section{B. Sensitivity to timing jitter}

In a previous paper [20], we looked at the sensitivity of the accelerator scheme to jitter on the timing between the laser and the injected electrons. For the laser intensity as chosen here, timing variations of order 100 fs (lowest currently reported [31]) could result in a large increase in energy spread and a decrease of the average energy. In order to show the ability of the compression scheme to compensate for timing jitter, the parameters of the example (Table I) have been chosen such as to accommodate a larger timing jitter without serious degradation of the beam quality. The results of variations in the timing between the laser and electron bunch can be seen in Fig. 5.

The results of Fig. 5 show that even large timing jitter of 1 ps has virtually no effect on the final energy and energy spread of the compressed bunch. The trade-off for this insensitivity is, as mentioned before, the longer length of the plasma channel and the lower final energy. If the expected jitter in the synchronization between laser and electrons is lower, the plasma channel length can be reduced.

\section{Tuning the compression factor}

If we want to change the compression factor of the plasma wave, there are two general ways to achieve this as can be seen from Eq. (1): changing the plasma density to change $\gamma_{g}$ or change the energy of the injected electrons $\left(\gamma_{0}\right)$. Here we will look at the effect of changing the plasma density of the compressor.

Changing the plasma density of the compressor also has several other effects: First, the amplitude of the plasma wave changes which means that either the laser power or the energy of the injected electrons has to be adjusted to ensure the electrons are still trapped. Second, the matched spot size of the plasma channel will change which means adjusting the laser spot size and power. Last, the dephasing length of the electrons in the plasma wave changes which means the total length of the plasma may need adjusting. To reduce the amount of simulations needed, we maintain the same wave amplitude and wavelength for the plasma wave, thus is no need to adjust the laser and electron parameters to still get (almost) the same amount of trapped electrons for different densities. Since according to 1D theory [Eq. (1)] the compression factor depends on the propagation speed of the plasma wave, we will adjust the speed of the plasma wave to that corresponding to the plasma density chosen. In this way, we are able to study the predicted effect of the change in plasma density/wave velocity without having to adjust the other electron, laser, and plasma parameters for each density. The result of varying the plasma density on the compressed bunch length can be seen in Fig. 6 .
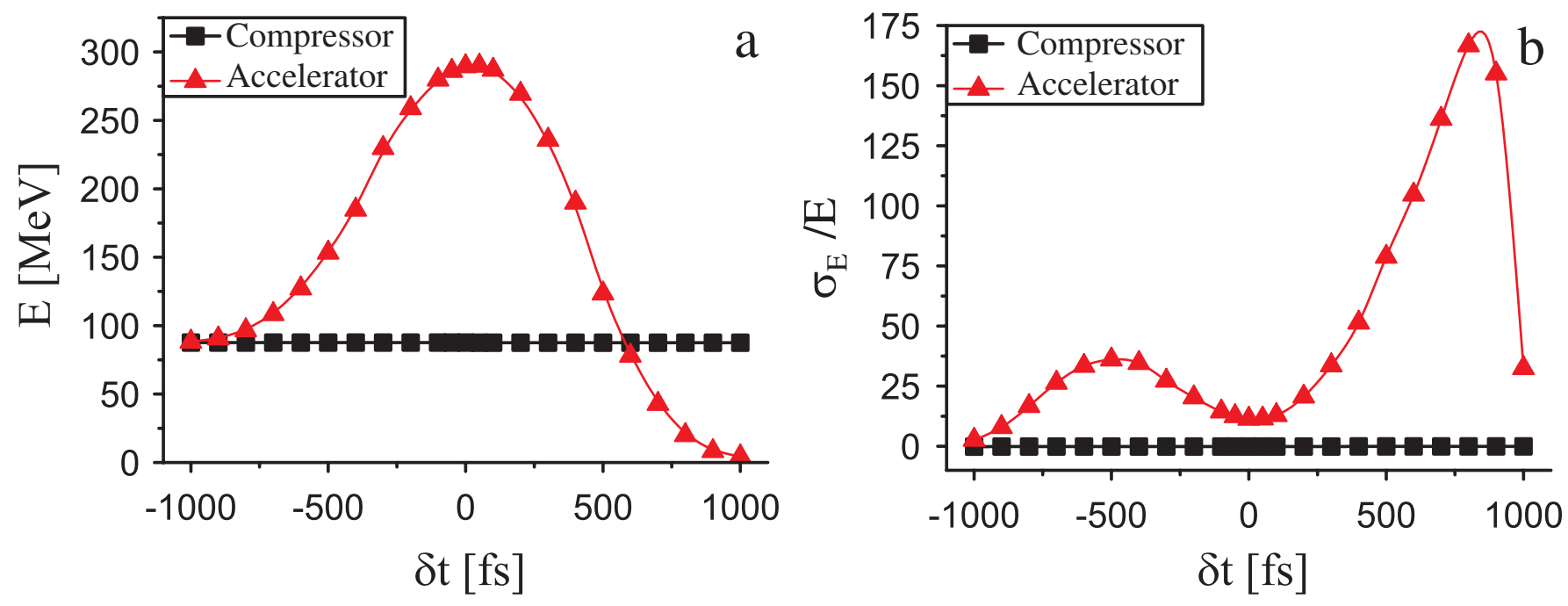

FIG. 5. (Color) Effect of fluctuations in the timing between laser and electron bunch on the energy (a) and energy spread (b) for both the compressor and accelerator scheme. 


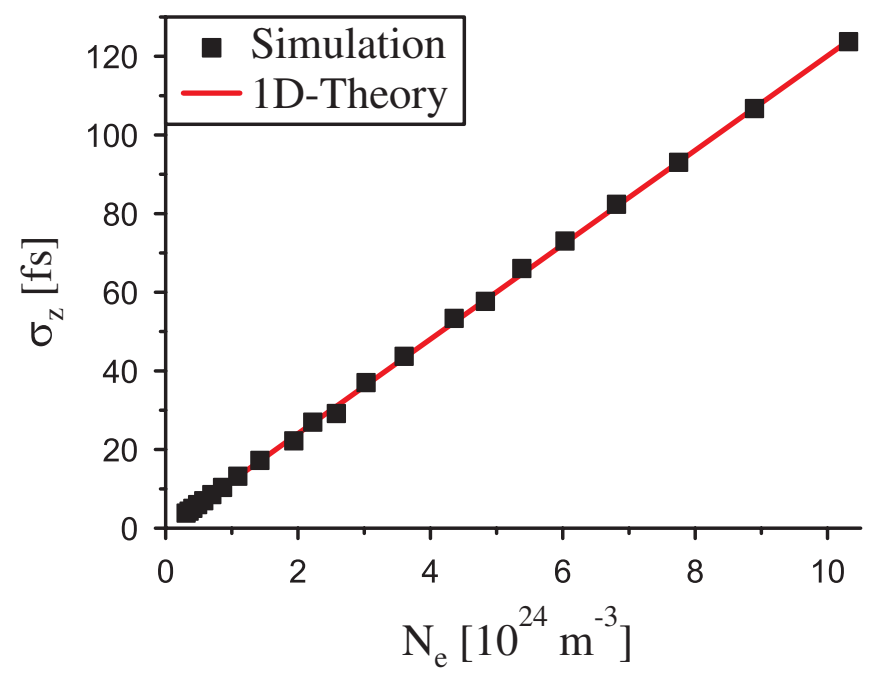

FIG. 6. (Color) Final bunch length of a 500 fs injected electron bunch after compression as a function of the density/propagation speed of the plasma wave. The solid line is the behavior predicted by 1D theory [Eq. (1)].

As can be seen from Fig. 6, the bunch length after compression corresponds well to the value predicted from 1D theory [Eq. (1)]. At lower densities, the dephasing length increases, but the chosen plasma channel is still long enough for the electrons to leave the plasma wave before exiting the plasma channel.

\section{Effect of the bunch length of the injected electrons}

When deriving the estimate for the compression factor of the scheme [Eq. (1) and [17]], it was (implicitly) assumed that the time needed to trap the electrons is much smaller than the time needed to accelerate them. When the injected electron bunches become long or the initial energy of the electrons is large, this assumption is no longer true. These conditions could therefore lead to less bunch compression. In Fig. 7 we see the final length of the electron bunch as a function of the length of the injected electron bunch for both the accelerator scheme and the compression scheme as well as the theoretical prediction.

As can be seen from Fig. 7, for short injected bunches $\left[L_{\text {bunch }}(\mathrm{FWHM}) \leq 500 \mathrm{fs}\right]$, the simulated results correspond very well to the theoretical prediction. However, for longer bunches, the final length of the bunches in the accelerator scheme becomes much larger than predicted. The cause of the lower compression can be seen in Fig. 8: the bunch is exiting the plasma before the entire injected bunch has been trapped. The length of the plasma is determined by the moment that the center of the original bunch leaves the accelerating phase of the plasma and enters the decelerating phase (moment of highest energy and lower energy spread). This effect can be partially compensated by using either lower energy electron bunches (and increasing the laser power to still get trapping) or by going to a lower plasma density, ensuring faster

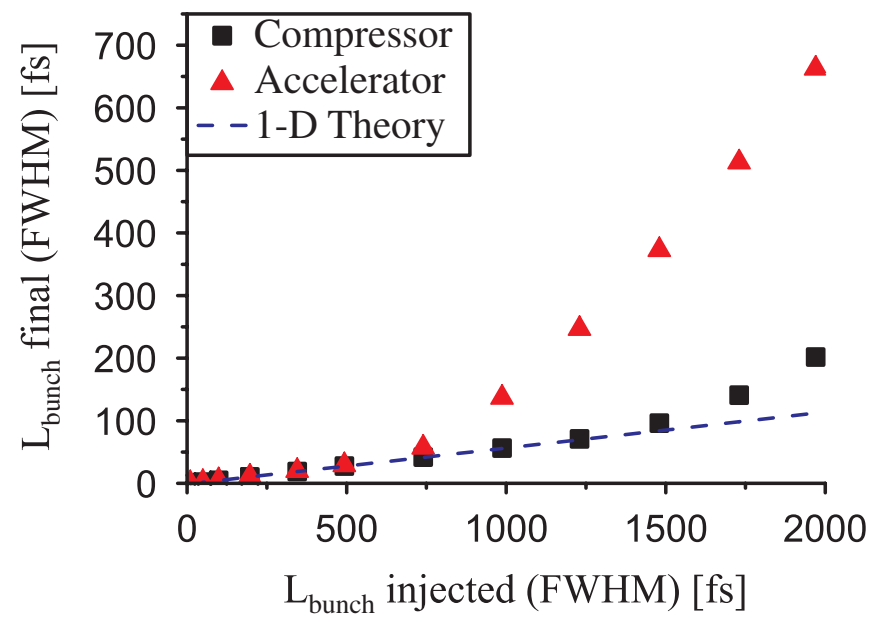

FIG. 7. (Color) Bunch length after compression as a function of the bunch length before compression for both the accelerator scheme and the compressor scheme compared to the theoretical compression of Eq. (1).

trapping/compression of the bunch. For the compression scheme the compression ratio remains as Eq. (1) predicts, except for very long bunches where part of the electrons are injected behind the laser pulse and get trapped there, separate from the main bunch, resulting in a longer apparent bunch length.

As can be seen from Fig. 8, the partial trapping and dephasing of long bunches in the accelerator scheme can lead to large energy spreads. Such a large energy spread will cause additional bunch lengthening and will also make the bunch unsuitable for most applications. An overview of the energy spread as a result of the length of the injected electron bunch can be seen in Fig. 9.

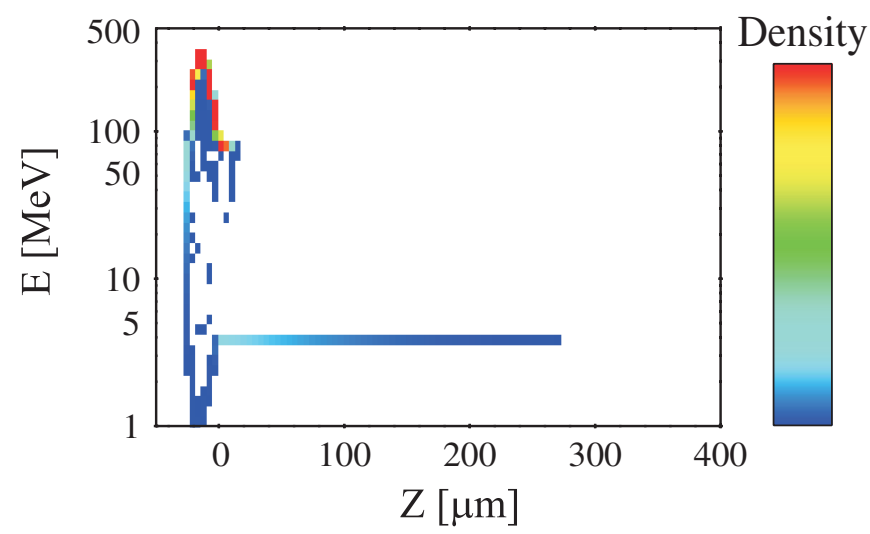

FIG. 8. (Color) Density plot of the position and energy of the electrons at the exit of the plasma channel in the bunch center of mass system (as in Fig. 1) for a bunch with an initial bunch length of $1250 \mathrm{fs}$ in the accelerator scheme. The center of the original bunch has reached the desired maximum energy at the exit of the plasma channel, but the front part of the bunch has not been trapped and compressed by the plasma wave yet and still retains it original energy and length. 


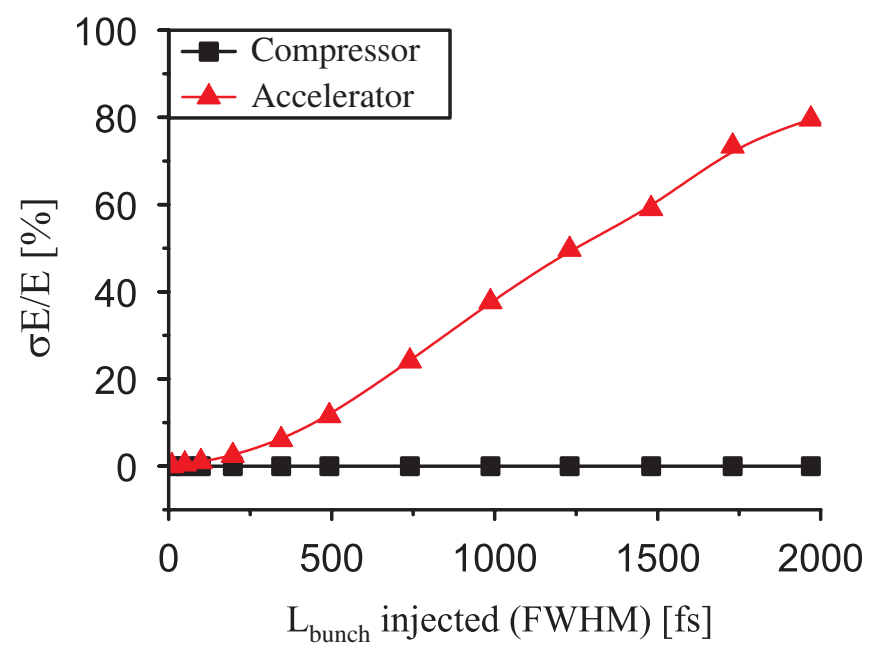

FIG. 9. (Color) Energy spread after compression as a function of the length of the injected bunch for both the compressor and accelerator scheme.

Figure 9 shows that the energy spread for the accelerator scheme rapidly rises for longer bunches (up to 80\%), while the final energy spread for the compression scheme remains constant (at $0.17 \%$ ).

\section{E. Effect of the energy spread in the injected bunch}

Thus far, all the injected electron bunches had a uniform energy. However, realistic electron bunches will always have an energy spread. This energy spread could lead to an increase in compressed bunch length and energy spread of the final beam since electrons with a different energy are trapped at a different phase of the plasma wave. Also, electrons with a lower energy might not get trapped by the plasma wave at all. To study the effect of energy spread in the injected bunch, we will first look at the resulting change in the bunch length after compression. These results can be seen in Fig. 10.

From the resulting compressed bunch lengths in Fig. 10, we can conclude the following: A small amount of initial energy spread $\left(\sigma_{E}<0.05 \mathrm{MeV}\right)$ in the injected electron bunch has little or no effect on the final bunch length. Energy spreads that are larger $(>1 \%)$ result in a linearly increasing final bunch length. This is consistent with trapping occurring in the linear part of the plasma wave as depicted in Fig. 1 as the trapping point varies linearly with the electron energy.

The effect of an initial energy spread in the injected bunch on the energy spread of the compressed bunch and the fraction of electrons trapped can be seen in Fig. 11.

As expected, the energy spread after compression increases with a larger initial energy spread of the injected electrons. The relative energy spread in the compressed bunch is however still smaller, even at maximum, than that of the injected bunch $\left(\frac{\sigma_{E}}{E}=4.1 \% \rightarrow \frac{\sigma_{E}}{E}=2.1 \%\right)$. The amount of trapped charge remains constant for injected

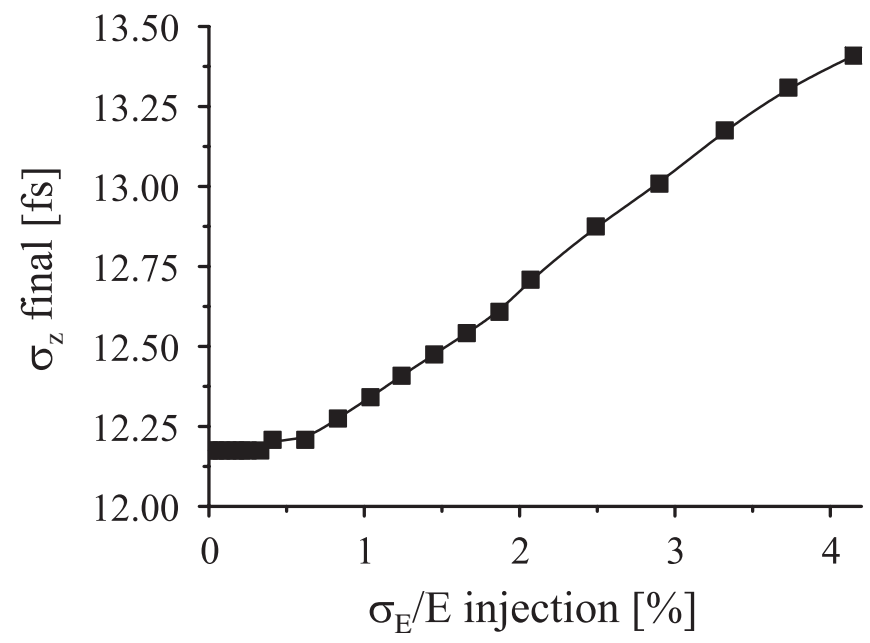

FIG. 10. Effect of the initial energy spread of injected electrons on the length of the compressed bunch.

bunches with a low initial energy spread $\left(\sigma_{E}<\right.$ $0.04 \mathrm{MeV}$ ), but for a larger initial energy spread, it quickly decreases until the trapped fraction is less than half of the amount trapped at low energy spread.

\section{F. The effect of space charge on the plasma compressor scheme}

As discussed in Sec. III, it is possible to include the effect of the space charge that the electron bunch exerts onto itself in the simulations at the cost of computational power needed. This has been done for compression using the standard parameters as given in Table I.

First we will look at the influence of space charge on the final bunch length after compression. The space-charge forces cause the electron bunch to expand. When the bunch is compressed, the charge density increases, causing an increased expansion. However, the electrons are also accelerated which causes a decrease in the effect of the

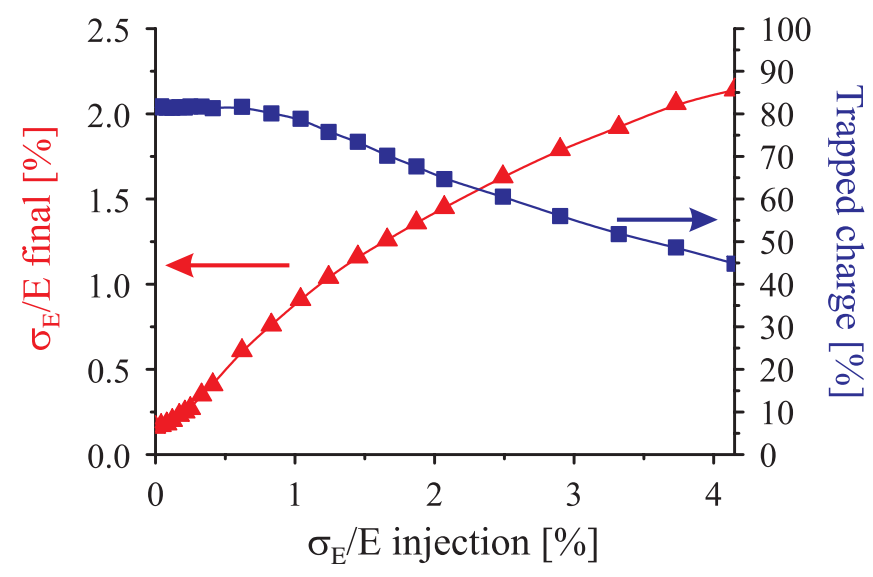

FIG. 11. (Color) Effect of the initial energy spread of injected electrons on the energy spread and trapped charge of the compressed bunch. 


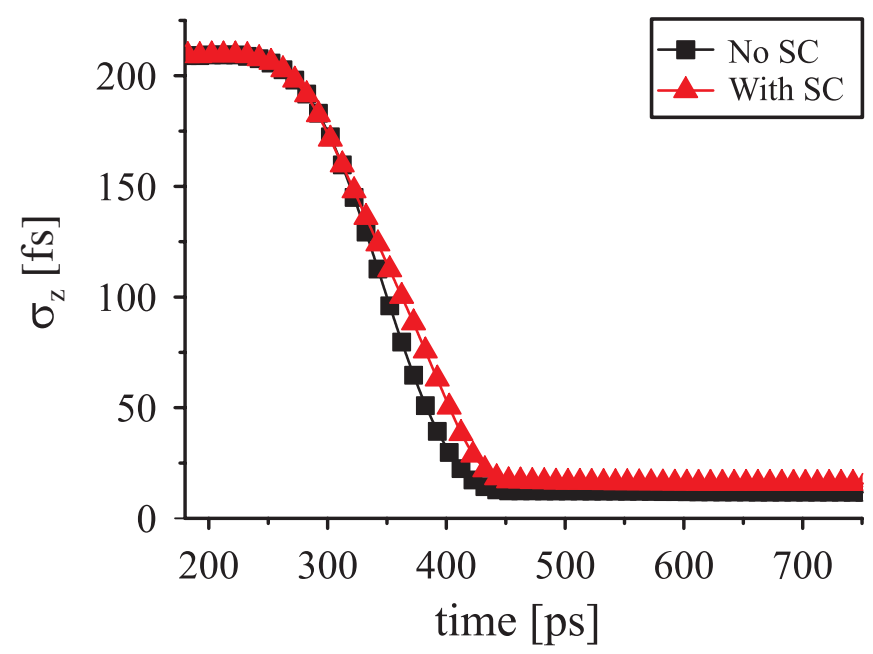

FIG. 12. (Color) Bunch length $\left(\sigma_{z}\right)$ of a $10 \mathrm{pC}$ injected electron bunch during compression with and without taking space charge into account.

longitudinal space-charge forces. The combined effect of space charge on the compression can be seen in Fig. 12.

As can be seen from Fig. 12, space charge causes a small decrease in compression for the electron bunch. Without taking space charge into account, the final compressed length is $12 \mathrm{fs}$, while with space charge the final length is $16 \mathrm{fs}$, both compared to the initial bunch length of $210 \mathrm{fs}$.

Another possible influence of space charge is a decrease of the final energy of the electrons, since the space-charge forces counteract the accelerating fields of the plasma wave working on the bunch. This effect can be seen in Fig. 13

Figure 13 shows that the space-charge forces do indeed decrease the acceleration of the electron bunch resulting in a lower maximum energy. However, the same forces also decrease the deceleration of the compressed electron bunch

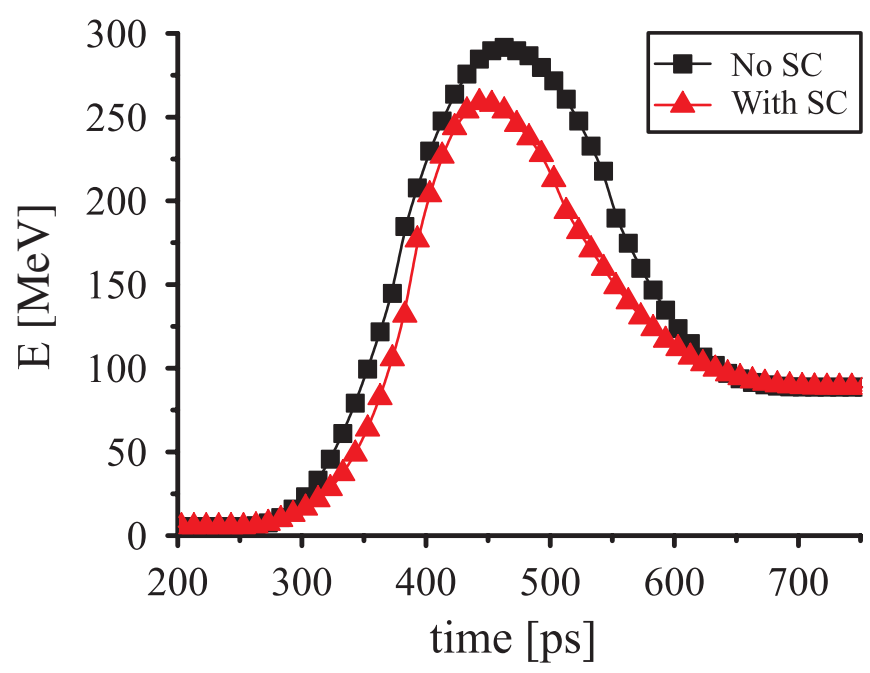

FIG. 13. (Color) Average energy $(E)$ of a $10 \mathrm{pC}$ injected electron bunch during compression with and without taking space charge into account. by the plasma wave resulting in the same final energy in both the simulations with and without space charge taken into account.

There is a decrease of the total amount of charge trapped when taking space-charge effects into account. This decrease is due to the radial expansion of the electron bunch caused by the space-charge forces. Because of this radial expansion, the radial size of the electron bunch is larger when the plasma wave catches up with the electrons as can be seen in Fig. 14. Since the fields in the plasma wave decrease in the radial direction, the electrons experience a lower trapping field, leading to a lower trapping. This effect can be (partially) canceled by increasing the radial overlap between laser and electron bunch by adjusting the focusing of the electron bunch into the plasma or increasing the radius of the laser (and adjusting the matched spot size of the plasma correspondingly). Alternatively, the laser/electron energy can be increased to increase trapping.

The longitudinal emittance is only slightly increased by the effects of space charge since the bunch is already relativistic before trapping and the forces exerted by the plasma wave are much larger than those of the spacecharge forces inside the bunch during most of the compression cycle. The influence of space charge on the longitudinal emittance can be seen in Fig. 15. The additional increase in emittance is mainly due to the electrons that have expanded in the radial direction before trapping due to space-charge induced radial expansion. These electrons will either be trapped in lower fields, leading to a lower energy, or will not be trapped by the plasma wave and continue their radial expansion until they hit the plasma wall (and are removed). After the removal of the untrapped electrons, the longitudinal emittance with space charge taking into account is almost equal to the emittance without space charge again.

The effect of space charge on the transverse emittance is much more pronounced than the effect on the longitudinal emittance as can be seen in Fig. 16. The reasons for the increase in emittance are again the same as in the longitudinal case, but they have much more effect on the transverse emittance. The primary effect of space charge is the radial expansion of the electron bunch; this increases the transverse size and energy spread of the bunch both of which cause an increase in the transverse emittance. The secondary effect is a decrease in trapping since the plasma wave at larger distances from the optical axis is weaker. This means that electrons either continue to expand radially or get focused to the optical axis, but then either overshoot or enter the defocusing phase of the plasma wave. Both these processes result in an increased radial velocity and corresponding increase in emittance. Once the electrons that have not been trapped have traveled far enough in the radial direction to be removed and all other electrons are trapped in the same phase of the plasma wave, the emittance decreases again. There is however still an 

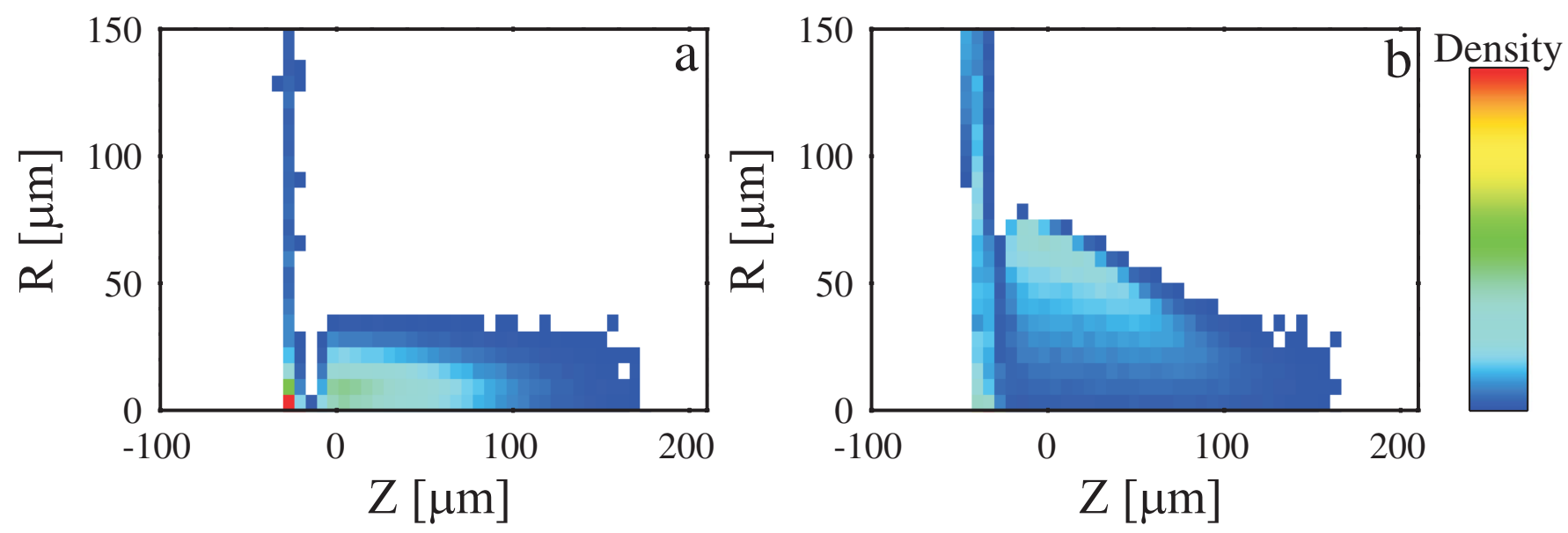

FIG. 14. (Color) Density plot of the radial profile of the bunch during trapping without (a) and with space charge (b).

increase in transverse emittance, both due to the space charge induced increase radial size of the bunch and the increase beam divergence (both are about 1.5 times as large as in the case with no space charge).

\section{G. The effect of initial bunch emittance on the plasma compressor scheme}

In most of the previous results, the initial bunch was assumed to possess zero emittance. We will now briefly discuss the effect of the initial bunch emittance on compression. The effects of a longitudinal emittance have already been discussed in Secs. IV D and IVE where the individual components (bunch length and energy spread) have been discussed. An initial longitudinal emittance can thus lead to a lower compression, higher energy spread, and a reduction of the amount of trapped charge.

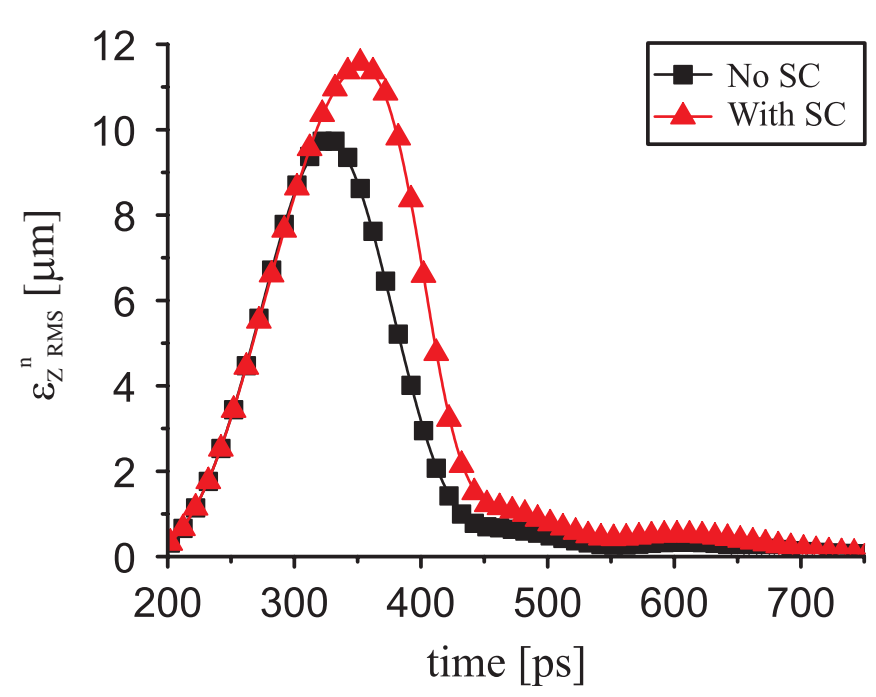

FIG. 15. (Color) Time evolution of the normalized RMS emittance in the longitudinal direction $\left(\varepsilon_{Z_{\mathrm{RMS}}}^{N}\right)$ with and without taking the effects of space charge into account.
The effect of the transverse emittance is highly dependent on the specific parameters of the electron bunch, plasma, and laser pulse: The scheme relies on the interaction between the plasma wave and the electron bunch. This means that, during the compression cycle, there must be a good overlap between the plasma wave and the electron bunch. A bunch with a large transverse emittance will have to be strongly focused in order to get it down to a small radial size. However, this means the bunch will also quickly defocus, possibly before the bunch has been completely trapped by the plasma wave. This leads to a decrease in trapped charge. Even at the focus, the larger radial velocity of the electron means the radial focusing of the plasma wave is less effective which again leads to lower trapped charge. To increased amount of trapped charge from a bunch with larger transverse emittance, there are two things that can be done: increase the trapping of the

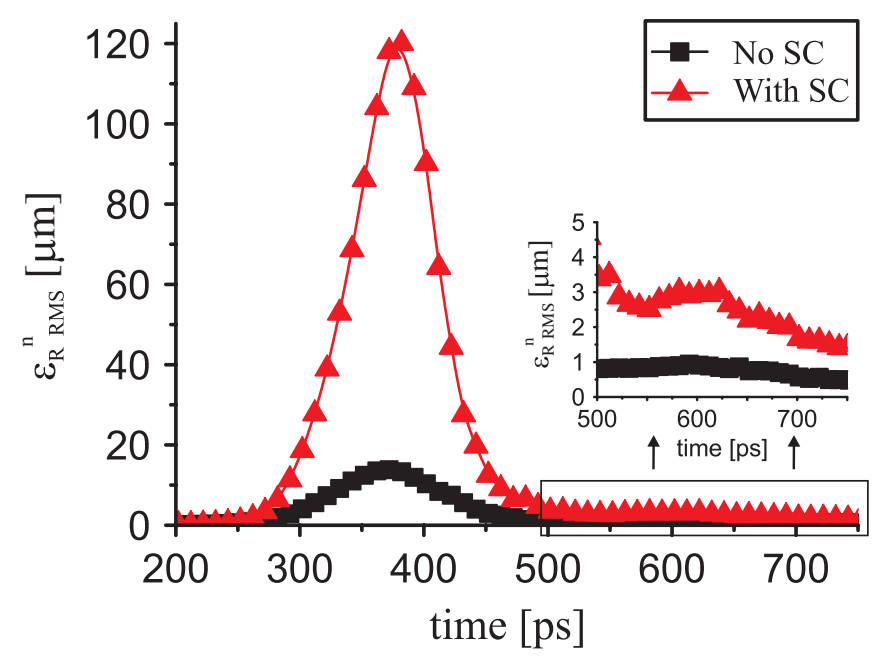

FIG. 16. (Color) Time evolution of the normalized RMS emittance in the transverse direction $\left(\varepsilon_{R_{\mathrm{RMS}}}^{N}\right)$ with and without taking the effects of space charge into account. The inset is an enlargement of the final part of the evolution. 
plasma wave and decrease the radial defocusing of the electron bunch during trapping. The trapping of the plasma wave can be increased by either increasing the trapping potential (increasing the laser power/plasma density) or increasing the electron energy (thus lowering the trapping potential needed). The radial defocusing of the electron bunch can be decreased in three ways: First, increase the radial size of the plasma wave by increasing the laser focus and adjusting the matched spot size of the plasma channel (combined with increasing the laser power to retain or increase the trapping potential), this increases the radial overlap. Second, decrease the focusing angle of the electron bunch. This results in a slower defocusing, but a larger focus, so it might have to be combined with an increase in plasma wave radius. Third, the trapping time can be decreased, allowing the bunch less time to defocus. This can be done by making the bunch shorter, decreasing the plasma density (and thus making the plasma wave faster), or by decreasing the electron energy (while increasing the laser power/plasma density to maintain sufficient trapping).

\section{CONCLUSIONS}

We have presented a novel scheme for using a laser wakefield in a plasma to compress and accelerate electron bunches. Compared to the acceleration scheme by Khachatryan $[17,18,21]$, this scheme sacrifices part of the energy gain to minimize the resulting energy spread, allow the compression of longer bunches and increase the stability with regards to timing fluctuations. Most importantly, it allows the compression of electron bunches typically produced in sources such as rf photoguns with an energy of several $\mathrm{MeV}$ and a bunch length of one or a few picoseconds to bunches with a length below $100 \mathrm{fs}$. The resulting compressed electron bunches have several desirable properties: short duration, low energy spread, high current density, and an energy sufficiently high to reduce spacecharge induced bunch lengthening. Moreover, the scheme does not involve off-axis deflection of the beam, conserving axial symmetry and preventing coherent synchrotron radiation induced instabilities [6].

The compressor scheme as presented here is largely insensitive to jitter in the synchronization between the laser and the electron bunch. By adjusting the plasma length and laser pulse delay, the amount of jitter that is present without degrading the beam quality can be changed.

The predictions from 1D theory [17] as given in Eq. (1) correspond well to the compression ratios found over a wide range of bunch lengths and plasma densities. It was shown that the plasma density can be used to control the compression ratio of the compressor, but changing the density will also require adjustments to the laser power, the spot size, and the plasma length.

The compression scheme also allows the efficient compression of relatively long bunches while still resulting in a compressed bunch with a low energy spread. This allows the use of electron sources which produce bunches with lengths exceeding $500 \mathrm{fs}$ without the increase in energy spread and decrease in compression encountered in the accelerator scheme [17].

We looked at the effect of energy spread in the injected electron bunches on the parameters of the compressed bunches. It was found that bunches with an energy spread below $0.04 \mathrm{MeV}(\sim 1 \%)$ only give rise to a small increase in the final energy spread without affecting the compression and trapping of the bunches. This requirement on the energy spread is routinely met by most modern electron sources used in accelerators.

Lastly, the effects of space charge and initial emittance in the injected bunch on the compression and energy spread were discussed as well as ways to prevent the decrease in trapped charge induced by higher space charge or initial emittance.

Concluding, the plasma compressor scheme presented in this paper offers the possibility of compressing low energy picosecond electron bunches to medium energy bunches with a duration below $100 \mathrm{fs}$ on a relatively short length scale and with only a small increase in absolute energy spread while the relative energy spread is reduced. By changing the plasma density and length and laser power, it is furthermore possible to adjust both the compression factor and the sensitivity for synchronization jitter between the laser and injected electron bunch.

\section{OUTLOOK}

One of the more interesting applications of the compression scheme described in this paper is the inclusion of the compressor in a conventional accelerator. In combination with the injector, it forms a compact source able to produce sub-100-fs bunches, with low energy spread and high current density, suitable for postacceleration to the desired energies or for direct usage.

\section{ACKNOWLEDGMENTS}

This work is part of the research program of the "Stichting voor Fundamenteel Onderzoek der Materie (FOM)," which is financially supported by the "Nederlandse Organisatie voor Wetenschappelijk Onderzoek (NWO)." We acknowledge the support of the European Community-New and Emerging Science and Technology Activity under the FP6 Structuring the European Research Area program (project EuroLEAP, Contract No. 028514).

[1] W. P. Leemans, C. G. R. Geddes, J. Faure, Cs. Tóth, J. van Tilborg, C. B. Schroeder, E. Esarey, G. Fubiani, D. Auerbach, B. Marcelis, M. A. Carnahan, R. A. Kaindl, J. 
Byrd, and M. C. Martin, Phys. Rev. Lett. 91, 074802 (2003).

[2] F. Grüner, S. Becker, U. Schramm, T. Eichner, M. Fuchs, R. Weingartner, D. Habs, J. Meyer-ter-Vehn, M. Geissler, M. Ferrario, L. Serafini, B. van der Geer, H. Backe, W. Lauth, and S. Reiche, Appl. Phys. B 86, 431 (2007).

[3] M. B. James, J. E. Clendenin, S. D. Ecklund, R. H. Miller, J. C. Sheppard, C. K. Sinclair, and J. Sodja, IEEE Trans. Nucl. Sci. 30, 2992 (1983).

[4] B. E. Carlsten and S. J. Russell, Phys. Rev. E 53, R2072 (1996).

[5] M. Biagini, M. Boscolo, M. Ferrario, V. Fusco, S. Guiducci, B. Spataro, C. Vaccarezza, M. Zobov, L. Serafini, R. Bartolini, G. Dattoli, L. Giannessi, L. Mezi, M. Quattromini, C. Ronsivalle, E. Chiadroni, P. Emma, and J. B. Rosenzweig, in Proceedings of the 2003 Particle Accelerator Conference, edited by J. Chew (IEEE, New York, 2003), Vol. 2, pp. 914-916.

[6] S. Heifets, G. Stupakov, and S. Krinsky, Phys. Rev. ST Accel. Beams 5, 064401 (2002).

[7] S. G. Anderson, J. B. Rosenzweig, P. Musumeci, and M. C. Thompson, Phys. Rev. Lett. 91, 074803 (2003).

[8] T. Tajima and J. M. Dawson, Phys. Rev. Lett. 43, 267 (1979).

[9] C. G. R. Geddes, C. Toth, J. van Tilborg, E. Esarey, C. B. Schroeder, D. Bruhwiler, C. Nieter, J. Cary, and W. P. Leemans, Nature (London) 431, 538 (2004).

[10] S.P. D. Mangles, C.D. Murphy, Z. Najmudin, A. G. R. Thomas, J. L. Collier, A.E. Dangor, E. J. Divall, P. S. Foster, J. G. Gallacher, C. J. Hooker, D. A. Jaroszynski, A. J. Langley, W. B. Mori, P. A. Norreys, F. S. Tsung, R. Viskup, B.R. Walton, and K. Krushelnick, Nature (London) 431, 535 (2004).

[11] J. Faure, Y. Glinec, A. Pukhov, S. Kiselev, S. Gordienko, E. Lefebvre, J.-P. Rousseau, F. Burgy, and V. Malka, Nature (London) 431, 541 (2004).

[12] W. P. Leemans, B. Nagler, A. J. Gonsalves, C. Toth, K. Nakamura, C. G. R. Geddes, E. Esaray, C. B. Schroeder, and C. J. Hooker, Nature Phys. 2, 696 (2006).

[13] D. F. Gordon, R. F. Hubbard, J. H. Cooley, B. Hafizi, A. Ting, and P. Sprangle, Phys. Rev. E 71, 026404 (2005).
[14] A. F. Lifschitz, J. Faure, V. Malka, and P. Mora, Phys. Plasmas 12, 093104 (2005).

[15] W. H. Urbanus, W. van Dijk, S. B. van der Geer, G. J.H. Brussaard, and M. J. van der Wiel, J. Appl. Phys. 99, 114501 (2006).

[16] W. van Dijk, S. van der Geer, M. van der Wiel, and G. Brussaard, Phys. Plasmas 15, 093102 (2008).

[17] A. G. Khachatryan, F. A. van Goor, K.-J. Boller, A. J. W. Reitsma, and D. A. Jaroszynski, Phys. Rev. ST Accel. Beams 7, 121301 (2004).

[18] A. G. Khachatryan, Phys. Rev. E 65, 046504 (2002).

[19] A. Khachatryan, M. Luttikhof, A. Irman, F. van Goor, J. Verschuur, H. Bastiaens, and K.-J. Boller, Nucl. Instrum. Methods Phys. Res., Sect. A 566, 244 (2006).

[20] W. van Dijk, J. M. Corstens, S. B. van der Geer, M. J. van der Wiel, and G. J.H. Brussaard, Phys. Rev. ST Accel. Beams 12, 051304 (2009).

[21] A. G. Khachatryan, JETP Lett. 74, 371 (2001).

[22] P. Volfbeyn, E. Esarey, and W. P. Leemans, Phys. Plasmas 6, 2269 (1999).

[23] P. Sprangle, E. Esarey, A. Ting, and G. Joyce, in Advance Accelerator Concepts, AIP Conf. Proc. No. 193 (AIP, New York, 1989), Vol. 193, pp. 376-387.

[24] F. Dorchies, J. R. Marquès, B. Cros, G. Matthieussent, C. Courtois, T. Vélikoroussov, P. Audebert, J. P. Geindre, S. Rebibo, G. Hamoniaux, and F. Amiranoff, Phys. Rev. Lett. 82, 4655 (1999).

[25] V. V. Ivanov, P.S. Antsiferov, K. N. Koshelev, M. R. Akdim, and F. Bijkerk, Phys. Rev. Lett. 97, 205007 (2006).

[26] L. G. N. E. Andreev and E. V. Chizhonkov, Russ. J. Numer. Anal. Math. Modelling 13, 1 (1998).

[27] T. A. J. P. Mora, Phys. Plasmas 4, 217 (1997).

[28] B. van der Geer and M. de Loos, Ph.D. thesis, Eindhoven University of Technology, 2001.

[29] A. Butler, D. J. Spence, and S. M. Hooker, Phys. Rev. Lett. 89, 185003 (2002).

[30] B. Broks, W. van Dijk, and J. J. A. M. van der Mullen, J. Phys. D 39, 2377 (2006).

[31] F. B. Kiewiet, A.H. Kemper, O. J. Luiten, G. J. H. Brussaard, and M. J. van der Wiel, Nucl. Instrum. Methods Phys. Res., Sect. A 484, 619 (2002). 